\title{
Effects of BDNF Val66Met Polymorphism on White Matter Microalterations of the Corpus Callosum in Patients with Panic Disorder in Korean Populations
}

\author{
Hyun-Ju Kim¹, Minji Bang', Kang Soo Lee', Tai Kiu Choi', Chun II Park ${ }^{1}$, and Sang-Hyuk Lee ${ }^{1,2} \bowtie$ \\ ${ }^{1}$ Department of Psychiatry, CHA Bundang Medical Center, CHA University School of Medicine, Seongnam, Republic of Korea \\ ${ }^{2}$ Department of Clinical Pharmacology and Therapeutics, CHA Bundang Medical Center, CHA University School of Medicine, Seongnam, \\ Republic of Korea
}

Objective The brain-derived neurotrophic factor (BDNF) Val66Met (rs6265) polymorphism is suggested to be associated with the pathophysiology of anxiety disorders, including panic disorder (PD). Although the fronto-limbic white matter (WM) microstructures have been investigated, the corpus callosum (CC) has not yet been studied regarding its relationship with BDNF Val66Met polymorphism in PD.

Methods Ninety-five PD patients were enrolled. The Neuroticism, the Anxiety Sensitivity Inventory-Revised, Panic Disorder Severity Scale, and Beck Depression Inventory-II (BDI-II) were administered. Voxel-wise statistical analysis of diffusion tensor imaging data was performed within the CC regions using Tract-Based Spatial Statistics.

Results The GG genotype in BDNF Val66Met polymorphism has significantly higher fractional anisotropy (FA) values of the body and splenium of the CC, neuroticism and depressive symptom scale scores than the non-GG genotype in PD. The FA values of the body of the CC in the two groups were significantly different independent of age, sex, neuroticism, and BDI-II.

Conclusion Our findings demonstrate that the BDNF Val66Met polymorphism is associated with WM connectivity of the body and splenium of the CC, and may be related to neuroticism and depressive symptoms in PD. Additionally, the CC connectivity according to BDNF polymorphism may play a role in the pathophysiology of PD.

Psychiatry Investig 2020;17(10):967-975

Key Words Panic disorder, Brain-derived neurotrophic factor, Corpus callosum, Neuroticism, Diffusion tensor imaging.

\section{INTRODUCTION}

Panic disorder (PD)-encompassing psychological, neurological, and biological dimensions-is an anxiety disorder characterized by predictive anxiety, unexpected panic attacks, and physical symptoms. These symptoms include sensations of shortness of breath, palpitation, or fear of dying. Recently, re-

\footnotetext{
Received: May 18, 2020 Revised: July 6, 2020

Accepted: August 5, 2020

$\triangle$ Correspondence: Sang-Hyuk Lee, MD, PhD

Department of Psychiatry, CHA Bundang Medical Center, CHA University School of Medicine, 59 Yatap-ro, Bundang-gu, Seongnam 13496, Republic of Korea

Tel: +82-31-780-5694, Fax: +82-31-780-5862, E-mail: drshlee@cha.ac.kr

$\triangle$ Correspondence: Chun Il Park, MD

Department of Psychiatry, CHA Bundang Medical Center, CHA University School of Medicine, 59 Yatap-ro, Bundang-gu, Seongnam 13496, Republic of Korea

Tel: +82-31-780-5694, Fax: +82-31-780-5862, E-mail: chunil1001@cha.ac.kr

(c) This is an Open Access article distributed under the terms of the Creative Commons Attribution Non-Commercial License (https://creativecommons.org/licenses/by$\mathrm{nc} / 4.0$ ) which permits unrestricted non-commercial use, distribution, and reproduction in any medium, provided the original work is properly cited.
}

search on neurobiological and genetic factors of PD has been emerged. Previously, several studies suggested that genetic factors might contribute to one's susceptibility of developing PD. The relative risk in first-degree relatives of proband with $\mathrm{PD}$ between 2.6 to 20 times higher. ${ }^{1}$ A number of twin studies have further demonstrated that over $40 \%$ of the identified liability toward PD can explain the heritable factors thereof., 2,3

The brain-derived neurotrophic factor (BDNF) protein is encoded by a gene found in humans on chromosome 11 and is part of the neurotrophin family of nerve growth factor essential for neurodevelopment. In the brain, the BDNF effects the hippocampus, cortex, and basal forebrain-areas vital to memory, learning, and higher thinking. ${ }^{4}$ Single nucleotide polymorphisms (SNPs) of BDNF have been widely investigated across various psychiatry disorders, and there are significant associations between BDNF polymorphisms and susceptibility of schizophrenia, ${ }^{5}$ major depressive disorders, ${ }^{6}$ and Alzheimer's disease. ${ }^{7}$ In particular, rs6265 which produces an amino acid substitution from valine (Val) to methionine (Met) in the BDNF 
gene $^{8}$ is the most investigated SNP throughout the field of psychiatric. ${ }^{9}$

A previous study (of non-clinical samples) suggests that the GG (Val/Val) genotype of rs6265 in BDNF show increased trait anxiety as measured via the State Trait Anxiety Inventory compared to those of the GA (Val/Met) genotype. ${ }^{10}$ A meta-analysis of patients with anxiety disorders show that the rs6265 GG genotype results in increased neuroticism scale scores compared to A-carriers. ${ }^{11}$ Although there is a lack of previous findings regarding the association of rs6265 with anxiety-related traits in patients with $\mathrm{PD}$, some studies suggest that BDNF rs6265, in particular, plays a role in psychopathology-such as neuroticism or trait anxiety-in PD. ${ }^{12,13}$ A further study investigates the association between rs6265 and trait anxiety in individuals with PD, showing lower trait anxiety scores in those with the GG genotype of rs6265 than individuals of the GA/ AA genotype in the early onset PD group. ${ }^{13}$ The other study suggests that BDNF SNP rs6265 had an interaction effect with Catechol-O-methyltransferase gene (COMT) polymorphism to neuroticism scores and anxiety levels in patients with PD. ${ }^{12}$

So far, the amygdala, hippocampus, frontal cortex, and sensory cortex areas-as they relate to the fear network model of PD-has been studied extensively. ${ }^{14}$ Recently, however, it has been suggested that the white matter (WM) microalterations of the corpus callosum (CC) might be associated with visual memory-related processing and cognitive and affective functions ${ }^{15}$-functions that may be major components of PD patients with higher anxiety sensitivity in their visual dependence and in perceiving external threatening stressors. ${ }^{16}$ The CC is the largest WM tract in the brain, integrating information between the hemispheres so as to control recognition and emotional functions, ${ }^{17-19}$ and is specifically associated with attention, anxiety, and depressive symptoms. ${ }^{20}$ However, there are a limited number of studies on the association between the CC and pathophysiology, anxiety-related traits, and symptoms in patients with PD.

Further research investigating the association between the BDNF rs6265 polymorphism and brain structural abnormalities (including WM) has also emerged. Previous studies show that the BDNF rs6265 is associated with a reduction in hippocampal tissue (a key structure for the encoding of emotionally relevant data into memory) as well as with anxiety disorders ${ }^{21}$ and major depressive disorders. ${ }^{22}$ Besides the genetic knowledge of limbic structures, a recent study showed that the A-carriers of rs6265 in healthy adults have higher structural WM connectivity in the $\mathrm{CC}^{23}$ Interestingly, the association between BDNF rs6265 and WM lesions of the frontal CC is present in elderly patients with major depressive disorder. ${ }^{24}$ Thus, we considered the need for further research on the association between the BDNF rs6265 polymorphism and the WM connec- tivity of the CC in PD.

The aim of our study is to determine 1) whether the BDNF Val66Met polymorphism is associated with the WM connectivity of the CC in PD and 2) whether it is correlated with anxiety-related trait vulnerability and measures of symptom severities. We formulated the following hypotheses: 1) there would be differences of the WM connectivity in the CC between the GG genotype group and the non-GG genotype group; 2) that there would be gaps of trait vulnerability and symptom severities scale scores between the two groups; and 3) that WM connectivity in the CC would be associated with trait vulnerability and symptom severity measures.

\section{METHODS}

\section{Participants}

A total of 95 patients with PD participated in this study. Between January 2011 and May 2019, study participants were recruited from the pool of PD patients treated in the Department of Psychiatry at CHA Bundang Medical Center (Gyeonggi-do, Korea). All participants were between 17 and 64 years of age, right-handed, and of Korean descent. Individual and family histories of PD patients were collected through interviews.

Participants with PD-with or without agoraphobia-were diagnosed by practiced psychiatrists using the Structured Clinical Interview to evaluate DSM-VI-TR (as per the Diagnostic and Statistical Manual of Mental Disorders, Fourth Edition, Text Revision (DSM-IV-TR) Axis I Disorder). Only patients with primary PD were included. Secondary lifetime diagnoses included major depressive disorders (17 patients) and generalized anxiety disorder (10 patients). Exclusion criteria included any history of schizophrenia, intellectual disabilities, alcohol and substance abuse or dependence, and current or past serious medical disorders-including neurological disorders, pregnancy, and contraindications to brain magnetic resonance imaging (MRI) scanning.

At the time of MRI acquisition, $78 \mathrm{PD}$ patients were undergoing pharmacotherapy with selective serotonin reuptake inhibitors (SSRIs), including escitalopram, paroxetine, and sertraline $[n=78$; escitalopram equivalence dosage, mean $( \pm S D)$ $9.33 \pm 7.48 \mathrm{mg} /$ day], and benzodiazepines as anxiolytics, including lorazepam, alprazolam, clonazepam and diazepam $[\mathrm{n}=88$; lorazepam equivalence dosage mean $( \pm S D) 1.92 \pm 1.96 \mathrm{mg} /$ day]. Brain MRIs were performed 11.55 \pm 24.11 days after the initiation of medication uptake. All patients with PD were undergoing pharmacotherapy with antidepressants and anxiolytics according to the Korean Mediation Algorithm for PD ${ }^{25}$ or Clinical Practice Guidelines: Treatment of PD. ${ }^{26}$

All study procedures conform to the Institutional Review Board regulations and the principles of Good Clinical Prac- 
tice delineated by the CHA Bundang Medical Center. After a sufficient explanation of the study was given to the participants, written informed consent was obtained from each participant (2019-05-030, 2018-06-029, 2011-11-164).

\section{Clinical assessments}

The Neuroticism-Extraversion-Openness (NEO)-neuroticism Inventory ${ }^{27}$ and the Korean version of the Anxiety Sensitivity Inventory-Revised (ASI-R) ${ }^{28,29}$ measurements were used at an early stage to evaluate potential trait markers for PD. The NEO-neuroticism inventory contains a total of 12 items and has a high internal consistency, ranging from 0.68 to $0.86 .^{30}$ Meanwhile, the ASI-R is the most commonly-used measure of sensitivity to anxiety and consists of the following items: fear of respiratory symptom; fear of cardiovascular symptom; fear of a publicly observable anxiety reaction; and fear of cognitive dyscontrol. The internal consistency coefficient of the Korean version of the ASI-R is 0.92 and its test-retest reliability is measured to be 0.82 .

The Panic Disorder Severity Scale (PDSS), ${ }^{31}$ Beck Depression Inventory-II (BDI-II), ${ }^{32}$ and the Beck Anxiety Inventory $(B A I)^{33}$ were used to measure the clinical severity of the participants' anxiety and depression symptoms as they are manifested at an early stage.

Additionally, only supportive psychotherapy was allowed during the study period. The therapeutic response to pharmacology was measured twice: once at 8 weeks and again at 1 year. These measures are defined as $40 \%$ of the total PDSS score reduction compared to the baseline scores..$^{34-36}$

\section{Genotyping}

To analyze the BDNF Val66Met (rs6265) polymorphism, Genomic DNA was extracted from peripheral whole blood samples using a G-DEXTM II Genomic DNA Extraction Kit per manufacturer's protocol (Intron Biotechnology, Korea). The detection of functional SNPs in the genes encoding BDNF was based on an analysis of primer extension products as generated out of the genomic DNA, and which has been amplified using a chip-based matrix-assisted laser desorption/ionization time-of-flight (MALDI-TOF) mass spectrometry platform (Sequenom Inc., San Diego, CA, USA). The primer sequence for BDNF Val66Met was 5'-ACG TTG GAT GCT TCA TTG GGC CGA ACT TTC-3'. The general procedures were performed according to the manufacturer's standard protocol. ${ }^{37}$ Details of the genotyping procedures have been described above.

The genotype frequencies of BDNF Val66Met for Asian populations have been reported as follows: ${ }^{38}$ Korean, AA (Met/ Met) genotype 23\%, GA (Val/Met) genotype 46\%, GG (Val/ Val) genotype $31 \%$. We grouped PD patients into GG and nonGG groups, the reasons are as follows: first, the BDNF Val66Met polymorphism genotypes were grouped across Met/Met and $\mathrm{Met} / \mathrm{Val}$ in accordance with much of the literature, as the homozygous Met/Met genotype is relatively smaller across populations; Secondly, previous studies show well-validated genotype grouping along GG and non-GG groups. ${ }^{10,39,40}$

\section{Image acquisition}

Magnetic resonance images were acquired using a 3 Tesla scanner (SignaHDxt, GE Healthcare, Milwaukee, WI, USA). Diffusion-weighted imaging (DWI ${ }^{41}$ was performed using an echo planar imaging (EPI) sequence using the following parameters: repetition time (17,000 ms), echo time (108 ms), field of view $(240 \mathrm{~mm})$, acquisition matrix $(144 \times 144)$, slice thickness $(1.7 \mathrm{~mm})$, and voxel size $(1.67 \times 1.67 \times 1.7 \mathrm{~mm}){ }^{3,42}$ We use a double-echo option to decrease eddy current-related distortions. To reduce the effects of EPI spatial distortions, we made use of an 8-channel coil and array of spatial sensitivity encoding (SENSE), sped-up by factor of 2 . Seventy axial slices-parallel to the anterior commissure-posterior commissure line encompassing the entire brain-were obtained in fifty-one directions with $b=900 \mathrm{~s} / \mathrm{mm}^{2}$. Eight baseline scans with $b_{0}=0 \mathrm{~s} /$ $\mathrm{mm}^{2}$ were performed. Diffusion tensor images (DTIs) were measured from DWI utilizing the least-squares method (approximate scan time of 17 minutes).

\section{Tract-based spatial statistics}

Voxelwise statistical analysis about fractional anisotropy (FA) data was performed using Tract-Based Spatial Statistics (TBSS) version 1.2, as implemented via the Functional Magnetic Resonance Imaging of the Brain (FMRIB) Software Library (FSL version 4.1, Oxford, UK, https://fsl.fmrib.ox.ac.uk/fsl) according to the standard procedure. ${ }^{43}$ The FA is a measure of the extent of an ellipsoid, and provides information about the degree of anisotropy in the voxel. First, the DTI preprocessing-which included skull stripping using the Brain Extraction Tool and eddy current correction-was performed using the FMRIB Software Library (FSL).$^{44}$ We used the FMRIB's Nonlinear Image Registration Tool to align all subjects' FA data into the standard space (Montreal Neurologic Institute 152 standard). All transformed FA images were then integrated and applied to the original FA map so as to create a standard-space version thereof. All transformed FA images were averaged to produce a mean FA image, which was then skeletonized to make a mean FA skeleton which only considered the center of the WM tracts. The threshold of the skeleton was set to FA>0.2 (the TBSS default) so as to only contain major fiber bundles.

To compare DTI indices [FA, mean diffusivity (MD), axial diffusivity (AD), and radial diffusivity (RD)], FSL FA images were used in order to achieve nonlinear registration and obtain stages of skeletonization, and to estimate the projection 
vectors from each individual subject onto the mean FA skeleton. ${ }^{45}$ The nonlinear warps and skeleton projection were applied to other images.

Mean FA skeletons were multiplied using the Johns Hopkins University DTI-based probabilistic tractography atlas, containing the $\mathrm{CC}^{46}$

\section{Statistical analysis}

We performed a voxel by voxel statistical analysis in order to detect regions of significant differences in the scores of the DTI indices (i.e., FA, MD, AD, and $\mathrm{RD}$ ) among genotype groups [the BDNF GG genotype group vs. non-GG (GA/AA) genotype group], respectively. We conducted this analysis using nonparametric permutation tests with a correction for multiple comparisons with the FSL Randomize program. In addition, analysis of covariance (ANCOVA) and multiple linear regression analysis were conducted making use of age, sex, intracranial volume (ICV), neuroticism, and BDI-II as covariates. This allowed us to confirm the effect of other variables upon the results.

To confirm the voxel-wise analysis results, the DTI indices of the CC were extracted; the indices demonstrated significant differences in the FA, MD, and RD values between the two groups. These results were applied to further analysis. We conducted correlation analyses to investigate whether regional differences in the FA value could potentially be associated with the variance in clinical symptom ratings among in each genotype group.

We performed statistical analyses using SPSS version 26.0 (IBM Corporation, Armonk, NY, USA). Differences with $\mathrm{p}<$
0.05 were statistically significant.

\section{RESULTS}

\section{Genotyping}

We divided the participants with PD into either a GG genotype or a non-GG genotype (A-allele carrier) groups of the $B D N F$ Val66Met polymorphism, in order to evaluate the differences between the groups' WM connectivity in their CCs.

The genotype distributions of all 95 patients with PD (GG: $n=25, G A: n=53, A A: n=17)$ were in accordance with the Hardy-Weinberg equilibrium $\left(\chi^{2}=1.46, \mathrm{df}=1, \mathrm{p}=0.23\right)$. Upon division of the participants with PD into the two aforementioned groups, they were not significantly different in terms of sex ( $\mathrm{p}=$ $0.11)$ or age $(\mathrm{p}=0.72)$ (Table 1$)$.

\section{Comparisons of trait vulnerability and symptom severity measures between the GG genotype group and non-GG genotype group with panic disorder}

The GG genotype group showed significantly higher scores the NEO-neuroticism, and BDI-II scales. However, there was no significant difference via the ASI-R and its subscales (Table 2).

\section{Comparison of the diffusion tensor imaging indices of the corpus callosum between the GG genotype group and non-GG genotype group with panic disorder}

Increased FA values in the posterior part of body and splenium of the CC were found in participants with the GG genotype compared to the non-GG genotype participants. How-

Table 1. Comparison of the sociodemographic and clinical characteristics between BDNF genotype groups in panic disorder

\begin{tabular}{|c|c|c|c|c|c|}
\hline & $\begin{array}{c}\mathrm{GG}(\mathrm{Val} / \mathrm{Val}) \\
\text { genotype group } \\
(\mathrm{N}=25)\end{array}$ & $\begin{array}{c}\text { Non-GG (Met-carriers) } \\
\text { genotype group } \\
(\mathrm{N}=70)\end{array}$ & $\mathrm{t}$ & $\mathrm{df}$ & $\mathrm{p}$ \\
\hline Sex, male/female (N) & $7 / 18$ & $32 / 38$ & 1.62 & 46 & 0.11 \\
\hline Age at scan (years, mean \pm SD) & $36.28 \pm 13.65$ & $37.26 \pm 11.02$ & -0.36 & 93 & 0.72 \\
\hline Education (years, mean $\pm S D$ ) & $12.88 \pm 2.97$ & $13.61 \pm 2.99$ & -1.04 & 91 & 0.30 \\
\hline Intracranial volume (mL, mean $\pm \mathrm{SD}$ ) & $1502.406 \pm 104.84$ & $1505.82 \pm 131.28$ & -0.08 & 47 & 0.94 \\
\hline Agoraphobia, yes (\%) & $13(56.5)$ & $46(71.9)$ & 1.28 & 35 & 0.21 \\
\hline Major depressive disorder, yes (\%) & $5(20.0)$ & $12(17.1)$ & 0.32 & 93 & 0.75 \\
\hline Generalized anxiety disorder, yes (\%) & $4(16.0)$ & $6(8.5)$ & 0.91 & 34 & 0.37 \\
\hline Duration of illness (months, mean \pm SD) & $62.28 \pm 48.99$ & $50.89 \pm 35.73-$ & 1.24 & 93 & 0.22 \\
\hline Kinds of SSRI \{escitalopram [N (\%)]/praoxetine $[\mathrm{N}(\%)]\}$ & $9(36.0) / 10(40.0)$ & $24(34.3) / 30(42.9)$ & & & \\
\hline SSRI equivalent dosage (mg, mean $\pm \mathrm{SD})^{*}$ & $11.08 \pm 10.27$ & $7.96 \pm 7.98$ & 1.55 & 96 & 0.13 \\
\hline Kinds of benzodiazepine $\{$ alprazolam $[\mathrm{N}(\%)] /$ clonazepam $[\mathrm{N}(\%)]\}$ & $22(88.0) / 6(24.0)$ & $55(78.6) / 15(21.4)$ & & & \\
\hline Benzodiazepine equivalent dosage $(\mathrm{mg}, \text { mean } \pm \mathrm{SD})^{\dagger}$ & $2.10 \pm 1.93$ & $1.64 \pm 1.86$ & 1.05 & 93 & 0.30 \\
\hline
\end{tabular}

*the approximate equivalent oral doses to $10 \mathrm{mg}$ Escitalopram are given, the approximate equivalent oral doses to $1 \mathrm{mg}$ Lorazepam are given. BDNF: brain-derived neurotrophic factor, SD: standard deviation, SSRI: selective serotonin re-uptake inhibitor 
Table 2. Comparison of trait vulnerability and symptom severity measures between BDNF genotype groups in panic disorder

\begin{tabular}{|c|c|c|c|c|c|}
\hline & $\begin{array}{c}\mathrm{GG}(\mathrm{Val} / \mathrm{Val}) \\
\text { genotype group }(\mathrm{N}=25)\end{array}$ & $\begin{array}{l}\text { Non-GG (Met-carriers) } \\
\text { genotype group }(\mathrm{N}=70)\end{array}$ & $\mathrm{t}$ & df & $\mathrm{p}$ \\
\hline Neuroticism total score (mean \pm SD) & $8.58 \pm 3.23$ & $6.80 \pm 3.74$ & 2.07 & 88 & $0.04^{*}$ \\
\hline ASI-R total score (mean $\pm S D)$ & $54.23 \pm 35.92$ & $48.71 \pm 22.96$ & 0.67 & 27 & 0.51 \\
\hline ASI-R fear of respiratory symptom & $20.13 \pm 12.64$ & $19.71 \pm 11.03$ & 0.15 & 84 & 0.88 \\
\hline ASI-R fear of publicly observable anxiety reaction & $11.22 \pm 9.06$ & $10.47 \pm 7.14$ & 0.40 & 85 & 0.69 \\
\hline ASI-R fear of cardiovascular symptom & $13.83 \pm 10.62$ & $12.38 \pm 7.80$ & 0.69 & 84 & 0.49 \\
\hline ASI-R fear of cognitive dyscontrol & $8.50 \pm 8.32$ & $6.02 \pm 5.78$ & 1.35 & 31 & 0.19 \\
\hline Initial PDSS total score (mean \pm SD) & $13.00 \pm 6.70$ & $12.77 \pm 6.34$ & 0.15 & 87 & 0.88 \\
\hline PDSS decrease (\%) at 8 weeks (mean \pm SD) & $2.11 \pm 45.21$ & $-2.73 \pm 172.00$ & 0.30 & 78 & 0.76 \\
\hline PDSS decrease (\%) at 1 year (mean \pm SD) & $6.57 \pm 73.21$ & $19.81 \pm 121.61$ & -0.19 & 68 & 0.85 \\
\hline BDI-II total score $($ mean \pm SD) & $21.17 \pm 13.04$ & $15.74 \pm 8.04$ & 2.37 & 88 & $0.02^{*}$ \\
\hline BAI total score $($ mean \pm SD) & $28.29 \pm 16.52$ & $25.63 \pm 12.52$ & 0.81 & 86 & 0.42 \\
\hline
\end{tabular}

${ }^{*} \mathrm{p}<0.05$. BDNF: brain-derived neurotrophic factor, SD: standard deviation, ASI-R: Anxiety Sensitivity Inventory-Revised, PDSS: Panic Disorder Severity Scale, BDI-II: Beck Depression Inventory-II, BAI: Beck Anxiety Inventory

ever, the $\mathrm{MD}$ and $\mathrm{RD}$ values showed inverse results from the FA values (Figure 1). Significance remained the same after controlling for age, sex, the presence of comorbidity, and intracranial volumes.

\section{Linear regression of BDNF Val66Met polymorphism on the fractional anisotropy values of body and} splenium of the corpus callosum with panic disorder

Although the age and sex of PD patients did not show any significant correlation or association with the FA of the body and splenium of the CC, we decided to include age and sex so as to control for the potential confounding factors apparent in the regression model. Results of the linear regression of the FA values of the body and splenium of the CC on BDNF Val66Met genotype in patients with PD are presented in Table 3. This analysis reveals an association of $B D N F$ Val66Met genotype and the FA values of the body $(\beta=-0.43, \mathrm{p}<0.001)$ and splenium of the $C C(\beta=-0.60, p<0.001)$. Specifically, the FA values of the body and splenium of the CC in the GG genotype group were higher than those in the non-GG genotype group after controlling for age, sex, neuroticism, and BDI-II scores.

\section{Exploratory correlation analysis of the diffusion tensor imaging indices of the corpus callosum and trait vulnerability and clinical symptom severity scores in the Val66Met genotype groups with panic disorder}

Correlation analysis between the WM regions in the body and splenium of the CC-where the FA values significantly differed, according to genotype between the two groups' trait vulnerability scores-reveals significant positive correlations between the FA values of the body of the CC regions, as well as the baseline ASI-R "fear of respiratory symptom" scores. In the GG genotype group, a positive and statistically significant correlation is shown, unlike in the non-GG genotype group $(\mathrm{r}=$ $0.249, \mathrm{p}=0.049$ ) (Figure 2).

\section{DISCUSSION}

To our knowledge, this is the first study to demonstrate that in patients with PD 1) the GG genotype group has significantly higher FA values in regard to the posterior part of the body and splenium of the CC, and 2) that the GG genotype group exhibits higher degree of neuroticism-a vulnerability trait of $\mathrm{PD}$-and depressive symptoms than patients in the non-GG genotype group. Furthermore, among the non-GG genotype group, PD patients with higher FA values in the body of the CC showed higher baseline ASI-R "fear of respiratory symptom" subscale scores. Our findings suggest that $B D N F$ Val66Met polymorphism is associated with WM connectivity of the body and splenium of the CC, possibly associated with neuroticism and depressive symptoms in patients with PD.

In the present study, we observe that measures of neuroticism are higher among patients in the GG genotype group. Our results are similar to those of a the previous study of heterogeneous anxiety disorders, that included PD, post-traumatic stress disorder, obsessive-compulsive disorder, phobic disorder, and generalized anxiety disorder. ${ }^{11}$ This similarity is evident in the observation that patients with PD in the GG genotype group display greater levels of neuroticism than those patients in the non-GG genotype group. ${ }^{11}$ Moreover, since neuroticism can be associated with depressive symptomatology, we also find more depressive symptoms in patients of the GG genotype group rather than those in the non-GG genotype group. 


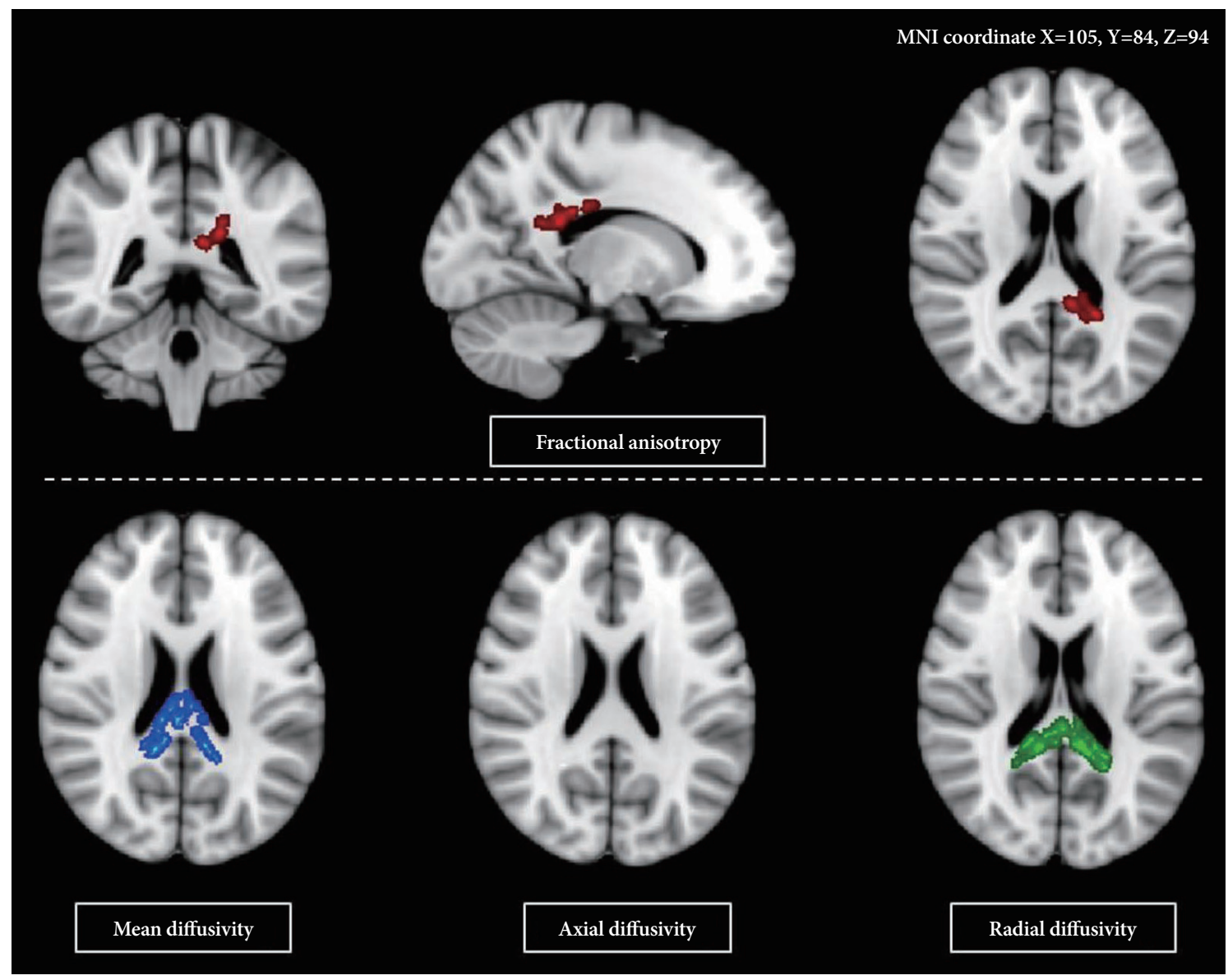

Figure 1. Results of the Tract-Based Spatial Statistics analysis show significant increases in fractional anisotropy (FA) values in the posterior part of the body and splenium of the corpus callosum in panic disorder patients of the GG genotype group compared to non-GG genotype group patients. Voxels demonstrating significantly (corrected $p<0.05$ ) increased FA values for the GG genotype group (compared to the non-GG genotype group) in patients with PD are shown in red. However, the mean diffusivity (MD) and radial diffusivity (RD) values show inverse results from the FA values. FA, MD, RD, and axial diffusivity (AD) are superimposed on the Montreal Neurologic Institute (MNI) $1 \mathrm{~mm}$ template. For better visibility, the results were thickened using the "tbss-fill" command. Red, Blue, and Green ( $p<0.05$; respectively; FEW corrected).

In this study, we further find that patients in the GG genotype group show higher FA values in the body and splenium areas of the $\mathrm{CC}$, as well as lower $\mathrm{MD}$ and $\mathrm{RD}$ values when compared to those in the non-GG genotype group. These findings suggest the possibility of a relationship between axon and myelin microalterations in the body and splenium regions of the CC of PD patients, according to the BDNF Val66Met polymorphism genotype. This may be evidence of WM abnormalities in PD patients, suggesting that microalterations in the WM contribute to the development of neuroticism and depressive symptoms in these patients.

The reason why the BDNF Val66Met polymorphism genotype is associated with the body and splenium areas of the CC is unknown. However, it has been suggested that the splenium of the CC can be considered a neocortical component of the hippocampal commissure, carriers fibers connecting the hippocampi with those linking the posterior parietal, medial temporal, and medial occipital cortices of the two hemispheres. ${ }^{47-49}$
In addition, in emotional disorders (e.g., depressive disorder), the BDNF Val66Met polymorphism is related to the WM connectivity of the CC, including the body and splenium regions thereof..$^{50}$ As depressive disorder can be comorbid with PD, and as PD can share the depressive symptomatology with depressive disorder as well, it is likely that the BDNF Val66Met polymorphism likely can affect the splenium of the CC connectivity in those with PD. However, our results as derived by multiple linear regressions show that the FA values of the body and splenium of the CC are higher than in those patients of the non-GG genotype group when controlling for age, sex, BDI-II, and neuroticism. This result supports our hypotheses that $B D N F$ Val66Met polymorphism can play an important role in explaining the microalterations of the body and splenium of the CC in PD patients regardless of age, sex, BDI-II, and neuroticism.

Literature regarding genetic-neuroimaging on $B D N F$ has investigated whether the Val66Met polymorphism influences 
WM abnormalities in healthy adults. ${ }^{51,52}$ In particular, previous results on BDNF Val66Met polymorphism in healthy adults present lower FA values in patients in the GG genotype group, contrary to our results of patients with PD. ${ }^{52}$ We explain this inconsistency as follows: first, certain studies suggest the microalterations of the CC's WM show higher FA values in patients with anxiety disorders, including PD. ${ }^{53-55}$ The second reason for these is unclear but may relate to differences in sampling and biotype grouping. Additionally, our sample was limited to Korean individuals, and certain previous studies which only include Caucasian individuals. ${ }^{52}$

Table 3. Linear regression of the FA values of body and splenium of corpus callosum on BDNF Val66Met genotype in panic disorder

\begin{tabular}{lccc}
\hline \multicolumn{4}{c}{$\begin{array}{c}\text { Dependent variable: FA values of splenium of corpus callosum } \\
\left(\chi^{2}=0.407, \mathrm{~F}=11.518, \mathrm{p}<0.000\right)\end{array}$} \\
\hline Variable & $\beta$ & $\mathrm{T}$ & $\mathrm{p}$ \\
\hline Age & 0.23 & 2.52 & $0.01^{*}$ \\
Sex & 0.10 & 1.14 & 0.26 \\
BDNF Val66Met genotype & -0.60 & -6.73 & $<0.000^{\dagger}$ \\
Neuroticism total score & 0.04 & 0.43 & 0.67 \\
BDI-II total score & 0.05 & 0.55 & 0.59 \\
\hline
\end{tabular}

Dependent variable: FA values of body of corpus callosum $\left(\chi^{2}=0.210, \mathrm{~F}=4.460, \mathrm{p}=0.001\right)$

\begin{tabular}{lccc}
\hline Variable & $\beta$ & $\mathrm{T}$ & $\mathrm{p}$ \\
\hline Age & 0.08 & 0.82 & 0.42 \\
Sex & -0.14 & -1.37 & 0.18 \\
BDNF Val66Met genotype & -0.43 & -4.18 & $<0.000^{\dagger}$ \\
Neuroticism total score & -0.02 & -0.16 & 0.87 \\
BDI-II total score & -0.09 & -0.79 & 0.43 \\
\hline
\end{tabular}

${ }^{*} \mathrm{p}<0.05,{ }^{\dagger} \mathrm{p}<0.001$. BDNF: brain-derived neurotrophic factor, FA: fractional anisotropy, BDI-II: Beck Depression Inventory-II
Our study also finds a positive association between the ASI$\mathrm{R}$ "fear of respiratory symptom" subscale score and the FA values in the body of the CC among patients in the non-GG genotype group. This result suggests that the WM connectivity of the body of the CC among PD patients in the non-GG genotype group might contribute to the trait vulnerability of anxiety sensitivity.

There were a number of limitations to our study. First, the sample size in each group was relatively small. Although significantly increased FA values in the CC regions were found among patients in the GG genotype group compared to the non-GG genotype group, further studies should include a larger sample for more accurate results. Secondly, only patients with PD were studied. To obtain the further understandings of the relationship between the BDNF Val66Met polymorphism and the CC of WM connectivity (such as genotype by diagnosis interaction), further studies should include a healthy control group. Third, the influence of medication on the WM connectivity in the CC across genotype groups could not be ruled out completely although the mean duration of medication day is relatively short.

In conclusion, the current findings suggest that the BDNF Val66Met polymorphism is associated with WM connectivity of the body and splenium of the CC, and that it may also be related to neuroticism and depressive symptoms in individuals with PD. Furthermore, PD patients in the non-GG genotype group, those with higher FA values in the body of the CC show higher anxiety sensitivity. These results demonstrate that the CC microalterations (according to the BDNF Val66Met polymorphism) are significant in the psychopathology of PD as depressive disorders.
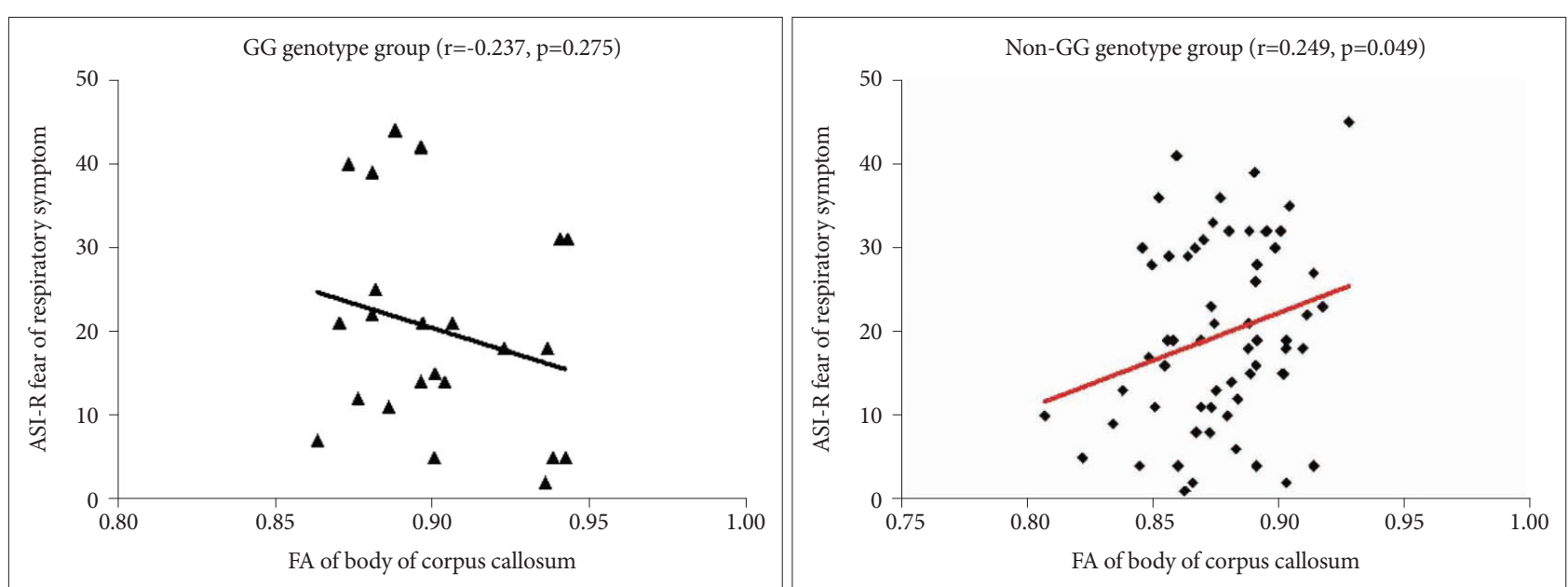

Figure 2. Significant positive correlations (corrected $p<0.05$ ) between the Anxiety Sensitivity Inventory-for Review only Revised (ASI-R) "fear of respiratory symptom" subscale score and fractional anisotropy (FA) values of the body of the corpus callosum in BDNF Val66Met polymorphism. 


\section{Acknowledgments}

This research was supported by the Basic Science Research Program through the National Research Foundation of Korea of funded by the Ministry of Education, Science and Technology (2011-0023359), by Ministry of Science and ICT (2018-R1D1A1B07046978) and (NRF-2019M3C7A1032262) to S.H. Lee. Thanks to Chaerim Song for the help of cluster analysis.

\section{Conflicts of Interest}

The authors have no potential conflicts of interest to disclose.

\section{Author Contributions}

Conceptualization: Hyun-Ju Kim, Chun Il Park, Sang-Hyuk Lee. Data curation: Hyun-Ju Kim, Chun Il Park, Sang-Hyuk Lee. Formal analysis: Hyun-Ju Kim, Chun Il Park, Sang-Hyuk Lee. Funding acquisition: SangHyuk Lee. Investigation: Hyun-Ju Kim, Chun Il Park, Sang-Hyuk Lee. Methodology: Hyun-Ju Kim, Chun Il Park, Sang-Hyuk Lee. Project administration: Hyun-Ju Kim, Chun Il Park, Sang-Hyuk Lee. Resources: Hyun-Ju Kim, Chun Il Park, Sang-Hyuk Lee. Software: Sang-Hyuk Lee. Supervision: Chun Il Park, Sang-Hyuk Lee. Validation: all authors. Visualization: Hyun-Ju Kim, Sang-Hyuk Lee. Writing_original draft: Hyun-Ju Kim, Chun Il Park, Sang-Hyuk Lee. Writing_review \& editing: Hyun-Ju Kim, Chun Il Park, Sang-Hyuk Lee.

\section{ORCID iDs}

Hyun-Ju Kim

Minji Bang

Kang Soo Lee

Tai Kiu Choi

Chun Il Park

Sang-Hyuk Lee https://orcid.org/0000-0002-8093-3366 https://orcid.org/0000-0002-1669-4014 https://orcid.org/0000-0001-6587-5623 https://orcid.org/0000-0003-0896-1925 https://orcid.org/0000-0003-0119-0443 https://orcid.org/0000-0001-7939-3000

\section{REFERENCES}

1. Crowe RR, Noyes R, Pauls DL, Slymen D. A family study of panic disorder. Arch Gen Psychiatry 1983;40:1065-1069.

2. Torgersen S. Genetics of neurosis. The effects of sampling variation upon the twin concordance ratio. Br J Psychiatry 1983;142:126-132.

3. Hettema JM, Neale MC, Kendler KS. A review and meta-analysis of the genetic epidemiology of anxiety disorders. Am J Psychiatry 2001;158: 1568-1578.

4. Yamada K, Nabeshima TJJops. Brain-derived neurotrophic factor/ TrkB signaling in memory processes. Am J Pharmacol Sci 2003;91: 267-270.

5. Xu MQ, St Clair D, Feng GY, Lin ZG, He G, Li X, et al. BDNF gene is a genetic risk factor for schizophrenia and is related to the chlorpromazine-induced extrapyramidal syndrome in the Chinese population. Pharmacogenet Genomics 2008;18:449-457.

6. Licinio J, Dong C, Wong ML. Novel sequence variations in the brainderived neurotrophic factor gene and association with major depression and antidepressant treatment response. Arch Gen Psychiatry 2009; 66:488-496.

7. Kunugi H, Ueki A, Otsuka M, Isse K, Hirasawa H, Kato N, et al. A novel polymorphism of the brain-derived neurotrophic factor (BDNF) gene associated with late-onset Alzheimer's disease. J Mol Psychiatry 2001;6:83-86

8. Egan MF, Kojima M, Callicott JH, Goldberg TE, Kolachana BS, Bertolino A, et al. The BDNF val66met polymorphism affects activity-dependent secretion of BDNF and human memory and hippocampal function. Cell 2003;112:257-269.

9. Buckley PF. Schizophrenia, An Issue of Psychiatric Clinics-E-Book. Elsevier Health Sciences; 2012.

10. Lang UE, Hellweg R, Kalus P, Bajbouj M, Lenzen KP, Sander T, et al. Association of a functional BDNF polymorphism and anxiety-related personality traits. Psychopharmacology 2005;180:95-99.
11. Frustaci A, Pozzi G, Gianfagna F, Manzoli L, Boccia SJN. Meta-analysis of the brain-derived neurotrophic factor gene (BDNF) Val66Met polymorphism in anxiety disorders and anxiety-related personality traits. Neuropsychobiology 2008;58:163-170.

12. Konishi Y, Tanii H, Otowa T, Sasaki T, Tochigi M, Umekage T, et al. Genex genex gender interaction of BDNF and COMT genotypes associated with panic disorder. Prog Neuropsychopharmacol Biol Psychiatry 2014;51:119-125.

13. Konishi Y, Tanii H, Otowa T, Sasaki T, Kaiya H, Okada M, et al. The association of BDNF val66met polymorphism with trait anxiety in panic disorder. J Neuropsychiatry Clin Neurosci 2014;26:344-351.

14. Gorman JM, Kent JM, Sullivan GM, Coplan JD. Neuroanatomical hypothesis of panic disorder, revised. Am J Psychiatry 2000;157:493-505.

15. Dalby R, Chakravarty M, Ahdidan J, Sørensen L, Frandsen J, Jonsdottir K, et al. Localization of white-matter lesions and effect of vascular risk factors in late-onset major depression. Psychol Med 2010;40:13891399.

16. Kim MK, Kim B, Choi TK, Lee SH. White matter correlates of anxiety sensitivity in panic disorder. J Affect Disord 2017;207:148-156.

17. Gazzaniga MS. Cerebral specialization and interhemispheric communication: does the corpus callosum enable the human condition? Brain 2000;123:1293-1326.

18. Quigley M, Cordes D, Turski P, Moritz C, Haughton V, Seth R, et al. Role of the corpus callosum in functional connectivity. AJNR Am J Neuroradiol 2003;24:208-212.

19. Walterfang M, Yücel M, Barton S, Reutens DC, Wood AG, Chen J, et al. Corpus callosum size and shape in individuals with current and past depression. J Affect Disord 2009;115:411-420.

20. Badaruddin DH, Andrews GL, Bölte S, Schilmoeller KJ, Schilmoeller G, Paul LK, et al. Social and behavioral problems of children with agenesis of the corpus callosum. Child Psychiatry Hum Dev 2007;38: 287-302.

21. Dincheva I, Lynch NB, Lee FS. The role of BDNF in the development of fear learning. Depress Anxiety 2016;33:907-916.

22. Verhagen M, Van Der Meij A, Van Deurzen P, Janzing J, Arias-Vasquez A, Buitelaar J, et al. Meta-analysis of the BDNF Val66Met polymorphism in major depressive disorder: effects of gender and ethnicity. Mol Psychiatry 2010;15:260-271.

23. Ziegler E, Foret A, Mascetti L, Muto V, Le Bourdiec-Shaffii A, Stender $\mathrm{J}$, et al. Altered white matter architecture in BDNF met carriers. PLoS One 2013;8:e69290.

24. Taylor WD, Zhao Z, Ashley-Koch A, Payne ME, Steffens DC, Krishnan RR, et al. Fiber tract-specific white matter lesion severity Findings in late-life depression and by AGTR1 A1166C genotype. Hum Brain Mapp 2013;34:295-303.

25. Kim MK, Lee JH, Kim M, Kim W, Moon E, Seo HJ, et al. Korean guidelines for the treatment of panic disorder 2018: initial and maintenance treatment strategies for the pharmacological treatment of panic disorder. Anxiety Mood 2018;14:53-62.

26. Bandelow B, Baldwin DS. Pharmacotherapy for Panic Disorder. In: Simon NM, Hollander E, Barbara O, Rothbaum BO, Stein DJ, Editors. The American Psychiatric Association Publishing Textbook of Anxiety, Trauma, and OCD-Related Disorders, Thrid Edition. Washington DC: American Psychiatric Pub, 2020, p.385-407.

27. Costa P, McCrae R. NEO Five-Factor Inventory (NEO-FFI). Odessa, FL: Psychological Assessment Resources 1989;3.

28. Lim YJ, Yu BH, Kim JH. Korean panic disorder severity scale: construct validity by confirmatory factor analysis. Depress Anxiety 2007; 24:95-102.

29. Taylor S, Cox BJ. An expanded anxiety sensitivity index: evidence for a hierarchic structure in a clinical sample. J Anxiety Disord 1998;12:463483.

30. Costa PT, McCrae RR. Normal personality assessment in clinical practice: The NEO Personality Inventory. Psychol Assess 1992;4:5-13.

31. Shear MK, Maser JD. Standardized assessment for panic disorder re- 
search: a conference report. Arch Gen Psychiatry 1994;51:346-354.

32. Beck AT, Steer RA, Brown GK. Beck Depression Inventory-II. San Antonio, TX: Psychological Corp; 1996.

33. Beck AT, Epstein N, Brown G, Steer RA. An inventory for measuring clinical anxiety: psychometric properties. J Consult Clin Psychol 1988; 56:893.

34. Barlow DH, Gorman JM, Shear MK, Woods SW. Cognitive-behavioral therapy, imipramine, or their combination for panic disorder: a randomized controlled trial. JAMA 2000;283:2529-2536.

35. Roy-Byrne PP, Russo J, Cowley DS, Katon WJ. Unemployment and emergency room visits predict poor treatment outcome in primary care panic disorder. J Clin Psychiatry 2003;64:383-389.

36. Shear MK, Brown TA, Barlow DH, Money R, Sholomskas DE, Woods SW, et al. Multicenter collaborative panic disorder severity scale. Am J Psychiatry 1997;154:1571-1575.

37. Yu ST, Kim MK, Kim B, Yoo E, Lee JY, Lee KS, et al. The effects of 5-HTR1A polymorphism on cingulum connectivity in patients with panic disorder. Psychiatry Investig 2013;10:399-406.

38. Pivac N, Kim B, Nedić G, Ho Joo Y, Kozarić-Kovačić D, Pyo Hong J, et al. Ethnic differences in brainderived neurotrophic factor Val66Met polymorphism in Croatian and Korean healthy participants. Croat Med J 2009;50:49-54.

39. Sen S, Nesse RM, Stoltenberg SF, Li S, Gleiberman L, Chakravarti A, et al. A BDNF coding variant is associated with the NEO personality inventory domain neuroticism, a risk factor for depression. Neuropsychopharmacol 2003;28:397-401.

40. Hünnerkopf R, Strobel A, Gutknecht L, Brocke B, Lesch KP. Interaction between BDNF Val66Met and dopamine transporter gene variation influences anxiety-related traits. Neuropsychopharmacol 2007;32: 2552-2560.

41. Hadwin JA, Garner M, Perez-Olivas G. The development of information processing biases in childhood anxiety: a review and exploration of its origins in parenting. Clin Psychol Rev 2006;26:876-894.

42. Mandelli L, Petrelli C, Serretti A. The role of specific early trauma in adult depression: a meta-analysis of published literature. Childhood trauma and adult depression. Eur Psychiatry 2015;30:665-680.

43. Smith SM, Jenkinson M, Johansen-Berg H, Rueckert D, Nichols TE, Mackay CE, et al. Tract-based spatial statistics: voxelwise analysis of multi-subject diffusion data. Neuroimage 2006;31:1487-1505.
44. Andersen SL, Tomada A, Vincow ES, Valente E, Polcari A, Teicher MH. Preliminary evidence for sensitive periods in the effect of childhood sexual abuse on regional brain development. J Neuropsychiatry Clin Neurosci 2008;20:292-301.

45. Bach M, Laun FB, Leemans A, Tax CM, Biessels GJ, Stieltjes B, et al. Methodological considerations on tract-based spatial statistics (TBSS). NeuroImage 2014;100:358-369.

46. Mori S, Aggarwal M. In vivo magnetic resonance imaging of the human limbic white matter. Front Aging Neurosci 2014;6:321.

47. Hofer S, Frahm J. Topography of the human corpus callosum revisited-comprehensive fiber tractography using diffusion tensor magnetic resonance imaging. Neuroimage 2006;32:989-994.

48. Saenz M, Fine I. Topographic organization of V1 projections through the corpus callosum in humans. Neuroimage 2010;52:1224-1229.

49. Aboitiz F, Montiel J. One hundred million years of interhemispheric communication: the history of the corpus callosum. Braz J Med Biol Res 2003;36:409-420.

50. Alexopoulos GS, Glatt CE, Hoptman MJ, Kanellopoulos D, Murphy CF, Kelly Jr RE, et al. BDNF val66met polymorphism, white matter abnormalities and remission of geriatric depression. J Affect Disord 2010;125:262-268.

51. Carballedo A, Amico F, Ugwu I, Fagan A, Fahey C, Morris D, et al. Reduced fractional anisotropy in the uncinate fasciculus in patients with major depression carrying the met-allele of the Val66Met brainderived neurotrophic factor genotype. Am J Med Genet B Neuropsychiatr Genet 2012;159:537-548.

52. Tost H, Alam T, Geramita M, Rebsch C, Kolachana B, Dickinson D, et al. Effects of the BDNF val 66 met polymorphism on white matter microstructure in healthy adults. Neuropsychopharmacol 2013;38:525532.

53. Kim B, Shin WS, Kim MK, Lee SH. White matter microstructural changes are associated with alcohol use in patients with panic disorder. J Affect Disord 2016;199:65-72.

54. Han DH, Renshaw PF, Dager SR, Chung A, Hwang J, Daniels MA, et al. Altered cingulate white matter connectivity in panic disorder patients. J Psychiatr Res 2008;42:399-407.

55. Ayling E, Aghajani M, Fouche JP, van der Wee N. Diffusion tensor imaging in anxiety disorders. Curr Psychiatry Rep 2012;14:197-202. 